\title{
AUC All Normalized by Weight
}

National Cancer Institute

\section{Source}

National Cancer Institute. AUC All Normalized by Weight. NCI Thesaurus. Code C92308.

The area under the curve (AUC) from the time of dosing to the time of the last

observation divided by the weight, regardless of whether the last concentration is measurable or not. 